\title{
Aníbal Núnez: Una relectura del tópico barroco de la ruina en el siglo veinte
}

\author{
Aníbal Núnez: A new reading of the baroque topic \\ of ruins in the twentieth century
}

\author{
Rocío Badía Fumaz \\ Universidad Complutense de Madrid. Madrid, España \\ rbadia@filol.ucm.es
}

\section{RESUMEN}

La tradición áurea recorre la obra de Aníbal Núñez, donde Góngora y Quevedo encuentran un espacio privilegiado. Este trabajo trata de enfrentar la utilización barroca del tópico de la ruina con su actualización en el siglo veinte por el poeta español Aníbal Núñez, destacando para ello tanto la pervivencia de rasgos estrictamente barrocos como las variaciones sobre el motivo que inevitablemente conlleva su incorporación en el contexto del siglo veinte. Se resaltará la concepción cíclica del tiempo, la identificación de la ruina con la casa y con el cuerpo, la esperanza de redimir el carácter destructivo de la ruina por la naturaleza, la sustitución del peregrino por el paseante y la conversión de la ruina en espacio sagrado y de conocimiento.

Palabras clave: Aníbal Núnez, Quevedo, ruina, tradición áurea, poesía siglo XX.

\section{ABSTRACT}

Golden Age Tradition has a great relevance in Aníbal Nuñez’s poetry, particularly Góngora and Quevedo's literature. This paper endeavours to compare the baroque treatment to ruins with the modern approach by Aníbal Núñez, focusing on the survival of baroque features and the innovations included. The most important loci will be the connection between ruins and home and body, the hopes of escaping from ruins through nature, the replacement of pilgrim by stroller and the ruin's change into a sacred space of wisdom.

Keywords: Aníbal Núnez, Quevedo, ruin, Golden Age Tradition, 20th century poetry.

Recibido: 07.03.2013. Aceptado: 15.09.2013 


\section{INTRODUCCIÓN}

Tacido en 1944 y fallecido en el año 1987, por tanto dentro de lo que 1 habitualmente se conoce como generación del 70 o generación del 68, Aníbal Núñez aparece como un poeta apartado del centro tanto por su misma especificidad física -inamovible de su Salamanca natal- como desde sus presupuestos poéticos, de un rigor e independencia casi absolutos, "obediente en todo momento a una desconexión y divergencia con el resto del cuerpo social" (Flor, 2008: 206-207). Poco difundidas, sus obras fueron apareciendo en brevísimas y ocasionales tiradas. Sólo en 1995 Fernando R. de la Flor y Esteban Pujals Gesalí logran ordenar un corpus poético disperso publicando en la editorial Hiperión su Obra Poética completa, que ha seguido enriqueciéndose desde entonces con nuevos manuscritos pero que sin duda contribuyó enormemente al conocimiento y estudio de este autor.

Tanto la obra de Aníbal Núñez como la de sus contemporáneos aparecen marcadas por una fuerte intertextualidad, donde la literatura del siglo de oro se convierte en fuente temática y lingüística privilegiada (Ferri Coll, 2004; Rozas, 1999: 305). Tal como la entendemos, esta tradición áurea se podría definir como la relectura y revisión de temas, motivos y tópicos lingüísticos que forman parte de la tradición literaria del siglo de oro y que son incorporados, con las correspondientes alteraciones, por un autor posterior en su propia obra. Es necesario recordar, como advierte Francisco J. Díez de Revenga, la originalidad que entraña este proceso: no tendría sentido "que se produjese una imitación servil de los clásicos admirados. Se trata de otra manera de entenderlos, de leerlos, de sentirlos y de reinterpretarlos" (2004: 110). Del conjunto de referencias intertextuales, la crítica ha señalado cómo, junto con la materia clásica, la literatura del siglo de oro español es motivo de continuas alusiones y reescrituras poéticas en la producción del poeta salmantino, tanto desde el contenido (Labrador, 2007; Herrero, 2005) como desde la formalización lingüística (Pardellas, 2009: 206; Casado, 2008: 84; García Valdés, 2008: 30; Piera, 2008: 20; Herrero, 2005: 325).

Pese a esta abundante referencialidad, entre la biblioteca personal que Aníbal Núñez dejó a su muerte se han conservado pocos volúmenes dedicados a la literatura de los siglos dieciséis y diecisiete, en comparación con la profusa intertextualidad de sus obras, pues apenas contaba con algunos ejemplares de Cervantes y de Calderón (Labrador, 2007), aun cuando la lectura de Quevedo, Góngora, Garcilaso, San Juan de la Cruz o Santa Teresa de Ávila coagula explícitamente a lo largo de su quehacer poético.

El tópico áureo de la ruina, que florece como un motivo temático cons- 
tante en la poesía de Aníbal Núnez, parece tener su origen en un soneto de Castiglione, conformándose, según José Lara Garrido y María Grazia Profeti, una cadena temática de modelos (ver, entre otros, Lara Garrido, 1980; López Bueno, 1986) que "incluye textos de Cetina, Herrera, Suárez de Figueroa, Arguijo, Francisco de Rioja, Medrano, Juan de Espinosa, los Argensola, el Conde de Villamediana” (Profeti, 2003: 714). Esta tradición terminará por conformar dos modelos de la ruina, renacentista y barroco, que pervivirán en la poesía posterior tanto en sus formas más puras como convergiendo en una única tradición. El modelo renacentista, centrado en la admiración y exaltación de un esplendor ya pasado, y el modelo barroco, orientado hacia el desengaño, encuentran acomodo en la poesía española del siglo veinte, en la cual acontece una clara revitalización del tópico de la ruina ${ }^{1}$.

Pero es especialmente el paradigma del tratamiento barroco de la ruina, tomado sobre todo de Quevedo, el que incide con mayor fuerza en la poesía de Aníbal Núnez; será este modelo el que guíe principalmente nuestro estudio, sin entrar a considerar la impronta que deja en el salmantino la poesía de ruinas del romanticismo inglés (Puppo, 2006) o las reflexiones sobre la historia de Walter Benjamin (Benéitez, 2010: 259), autores que también modelaron el imaginario de la ruina en Aníbal Núñez.

\section{EL TIEMPO DESDE LA RUINA}

La caracterización barroca de la ruina se construye sobre una llamativa polaridad. Por una parte, se rompe con el carácter extático que el ser humano reconoce en la piedra: ese simbolismo casi prehistórico que otorgaba a la piedra la cualidad de la inmutabilidad, de la perduración sobre la brevedad de lo vivo o sobre la variabilidad de otros elementos naturales (Revilla, 2007: 105) se va a teñir por vez primera de vulnerabilidad. La contemplación de la piedra erosionada va a conducir a una reflexión metafísica, en tanto que aquellas bases sólidas sobre las que el hombre se apoya-roca, civilización-se descubren frágiles, si no perecederas. La clave de este nuevo acercamiento

${ }^{1}$ María D. Martos Pérez, en su obra Las ruinas en la poesía española contemporánea. Estudio y antología (2008) establece una nómina provisional de autores que parte de la Generación del 27 con Cernuda, Guillén y Aleixandre, para continuar con Francisco Brines, Antonio Colinas, Guillermo Carnero, Pablo García Baena, Aníbal Núnez, Cesar Antonio Molina o Víctor Botas, entre otros. Otro tanto lleva a cabo Ángel L. Prieto de Paula en "La tradición literaria de las ruinas en los poetas del 70” (1991), destacando las obras de Guillermo Carnero, Antonio Colinas y Aníbal Núñez. 
simbólico a la piedra está en el proceso mismo que soporta: la erosión, acción destructora, está aliada con el tiempo. Frente a una devastación brusca, instantánea, producida por un agente de la realidad, la ruina no es sino roca sometida al tiempo; para reconocerla es necesaria una proyección hacia el pasado. Si al erigir un dolmen el hombre mira hacia el futuro, la ruina sólo puede reconocerse sobre la base de ese conocimiento anterior en el que la ruina era monumento, piedra recién erigida. Este conocimiento es el que permite adivinar la decadencia presente y anticipar la futura, causada por esa erosión aliada del tiempo, pues la perspectiva para reconocerla nos sitúa en la lejanía de la erección primera; una vida no permite, en su brevedad, apreciarla, sino que ha de mirarse por encima de generaciones previas ya desaparecidas.

Por otra parte, en las construcciones megalíticas la piedra se ha utilizado comúnmente como monumento funerario. De forma activa, la piedra se escoge por su perennidad para señalar la muerte, perennidad que puede contenerse en sí misma de manera simbólica -de nuevo, aparente triunfo del hombre sobre el tiempo (Revilla, 2007: 107) - pero que, sin duda alguna, se desdobla cuando la piedra es ruina: el monumento devastado por la erosión se convierte autorreferenciándose en su propia lápida, memoria de lo que fue y ya no es. Lo erigido es a la vez lápida -perennidad de la materia- y ruina -escombro del tiempo-.

Esta última significación es la que prefiere destacar en su Diccionario de símbolos, obra conocida por Aníbal Núñez (1995b: 157), Juan Eduardo Cirlot, para quien el sentido simbólico de las ruinas "es obvio y literal; significan destrucciones, vida muerta. Son sentimientos, ideas, lazos vividos que ya no poseen calor vital, pero que todavía existen, desprovistos de utilidad y función, en orden a la existencia y el pensamiento, pero saturados de pasado y de realidad destruida por el paso del tiempo. Las ruinas son un símbolo equivalente al de las mutilaciones en lo biológico" (2007: 396), definición donde la ruina supone el vaciamiento absoluto, la ausencia palpable.

\section{NUEVOS MATICES DEL DESENGAÑO BARROCO}

Aníbal Núñez recoge las tres interpretaciones de la ruina -antropológica, barroca y cirlotiana-, construyendo sobre ellas su propia reflexión, especialmente en los libros Definición de savia, Alzado de la ruina y Cuarzo. En una primera etapa la ruina va unida a la desesperanza consciente, al desengaño barroco, que tiene su manifestación paradigmática en el soneto de Quevedo 
"A Roma sepultada en sus ruinas", al condensar buena parte de los tópicos temáticos que tendrán gran relevancia en la pervivencia del motivo ${ }^{2}$. La unión de dos tiempos en un mismo espacio que mueve al sujeto a la reflexión - "Buscas en Roma a Roma, ¡oh peregrino!/ y en Roma misma a Roma no la hallas"-, constituye una unidad espacio-temporal muy fuerte, un cronotopo que aúna las claves de la consideración barroca de la ruina -desengaño, carácter implacable del tiempo, volubilidad de los bienes materiales, articulación de naturaleza y artificio-, visión que va a adensar sus significados al ir siendo utilizada por cada autor posterior en esa larga cadena donde "[a] cada movimiento innovador corresponde la disposición diferenciada hacia la realidad y el recurso a medios expresivos que conforme avanzamos en el siglo XVI se van complicando con la adherencia de los modelos previos cada vez más inducidos a convertirse en una cadena temática" (Lara Garrido, 1980: 386), extendiéndose hasta la más reciente poesía del siglo veinte.

De igual modo, a medida que la poesía de Aníbal Núñez va madurando, puede reconocerse en el salmantino una elaboración más personal del tópico, de forma que el primer carácter mutilado de la ruina defendido por Cirlot no va a ser, efectivamente, rebatido, pero ni mucho menos se va a admitir como símbolo conclusivo a la manera barroca. Sobre el peso de la ausencia que prima en el soneto de Quevedo, vamos a asistir a un enriquecimiento, a una superposición de los elementos naturales vivos que paulatinamente ocupan el espacio perdido de la piedra para crear un nuevo volumen más amplio. La pérdida primera permite el crecimiento posterior, la ampliación formal y de significado.

El tópico de la ruina en Aníbal Núñez supone también un enriqueci-

\footnotetext{
${ }^{2}$ Buscas en Roma a Roma, joh peregrino!, y en Roma misma a Roma no la hallas: cadáver son las que ostentó murallas, y tumba de sí proprio el Aventino.

Yace donde reinaba el Palatino; y limadas del tiempo, las medallas más se muestran destrozo a las batallas de las edades que blasón latino.
}

Sólo el Tibre quedó, cuya corriente, si ciudad la regó, ya, sepoltura, la llora con funesto son doliente.

¡Oh, Roma!, en tu grandeza, en tu hermosura, huyó lo que era firme, y solamente lo fugitivo permanece y dura (Quevedo, 1969: 418). 
miento desde el punto de vista del tratamiento temporal, donde a la unión de pasado y presente, que se juntan gracias a un único punto de vista privilegiado en Quevedo -desde el que se reconoce el cambio que el tiempo ha infligido en el espacio y se extrae desde el presente una reflexión metafísica-, el salmantino va a sumar una consideración de la ruina como proceso sin final, proyectada hacia el futuro no como estela conmemorativa sino como elemento vivo, cambiante. Veamos un primer ejemplo extraído del poema "Ruinas de San Fernando":

Seis arcos ciegos: resto ahora patente, ayer y luego oculto;

libre por el momento de lo que sea vivienda

y es edificación, vierte más hielo

sobre quien, aterido de esperanza, contempla tras la valla y diseña un futuro que irá desvaneciéndose (Núñez, 1995a: 311).

Los versos "resto ahora patente/ ayer y luego oculto" nos introducen en el carácter dinámico de la ruina. Ésta aparece y desaparece; surge precisamente de una desaparición previa, para convocar luego en el yo poético cierto deseo de recuperación de lo desaparecido, conformidad con la forma presente y certeza de su posterior degradación. El presente no es por tanto sólo el momento del desengaño, sino que es un presente desde el que se tienden líneas al pasado -ruina que fue y que en un futuro hipotético será- y al futuro con la apertura a los posibles usos que aún pueda tener la construcción, "libre por el momento de lo que sea vivienda". El "futuro/ que irá desvaneciéndose" no marca un final sino que la forma verbal elegida, "desvaneciéndose", quiere hacer también explícita esa continuidad hacia el futuro, aunque todavía la encontremos únicamente como pérdida.

A diferencia del poema de Quevedo, no se manifiesta ya sorpresa o lamento alguno en presencia de la ruina, no sobrecoge su contemplación. El yo poético es, en cierto sentido, poco ingenuo. La conciencia aguda de la ruina evita la sorpresa precisamente por medio del conocimiento: "Ve en la ruina la Ruina”, prosigue el poema. En palabras de Miguel Casado, "[1]a ruina, en realidad, preexiste a la misma ruina: es un sistema anterior, infiltrado en la inutilidad de toda lucha" (1999: 170) y "[e]s esta presencia el horror de la ruina; el horror es su antigüedad, su pertenencia al ser" (1999: 171), conciencia reforzada en el poeta por la extensa cadena literaria que arrastra el motivo, convertido ya en tópico. 


\section{LA CASA Y EL CUERPO}

El paso del tiempo aludido por la decadencia de lo perecedero -transformación de la ciudad en ruina, de la Roma primera en otra distinta- permite establecer un paralelismo entre el proceso de degradación de la piedra y el del ser humano, entre la ruina y el cuerpo (Orozco, 1947: 126), cuya presencia en el Quevedo de los poemas morales es evidente (Tobar, 2002: 250): "El cuerpo es edificio de la tierra,/ y en ella habemos de volvernos todos/ desatados en polvo, cuando el cielo,/ de tan vil edificio desceñidos/ reciba el alma, que en prisión de barro/ reinó en pobre república y enferma”. Estos versos del Phocílides encuentran un desarrollo mayor en el poema "Enseña cómo todas las cosas avisan de la muerte", donde la identificación casa-cuerpo se amplía añadiéndose la patria, también como sinónimo de casa, para enriquecer la relación por medio de la oposición de atributos ${ }^{3}$.

Siguiendo la identificación entre "patria" y "casa" propuesta por la crítica (Tobar, 2002) y la interpretación del soneto como reflexión sobre la muerte (Ferri Coll, 2010) podemos destacar la utilización de adjetivos del ámbito de lo humano para referirse a la casa: muros cansados, báculo menos fuerte, espada por la edad vencida. El choque entre la descripción de la casa por medio de sus atributos particulares - muros, habitación, báculo y espada- y los adjetivos utilizados crean la caracterización del sujeto poético. El soneto, pues, ha de entenderse casi exclusivamente como descripción de ese yo poético que, construido por medio de esos adjetivos metafóricos, reconocemos como cansado, anciano, encorvado, menos fuerte y por la edad vencido. María D. Martos observa acertadamente en Aníbal Núñez esta misma "co-

\footnotetext{
${ }^{3}$ Miré los muros de la patria mía, si un tiempo fuertes ya desmoronados, de la carrera de la edad cansados, por quien caduca ya su valentía.

Salíme al campo. Vi que el sol bebía los arroyos del hielo desatados, y del monte quejosos los ganados que con sombras hurtó su luz al día.

Entré en mi casa. Vi que, amancillada, de anciana habitación era despojos; mi báculo, más corvo y menos fuerte.

Vencida de la edad sentí mi espada, y no hallé cosa en que poner los ojos que no fuese recuerdo de la muerte (Rey, 1997: 201-202).
} 
rrelación entre las ruinas y el edificio de sangre que metaforiza el cuerpo humano, el hombre y su propia existencia metafísica" (2008: 44), aunque sin aludir a una probable herencia barroca.

Continuando con el soneto de Quevedo, el desmoronamiento, la destrucción material y vital, surge en medio de la equiparación entre la casa y el cuerpo para enfrentarse a una naturaleza plenamente armónica - "Vi que el sol bebía/ los arroyos del hielo desatados"- que permanece ajena en su completitud; tópico del beatus ille que "no se detiene en los atractivos rurales, ni conduce al amor, a la contemplación de los astros o a la visión de Dios. En su mayoría, las composiciones que cantan la aurea mediocritas ofrecen alguna forma de meditación sobre la muerte" (Rey, 1997: 189).

La posibilidad de concebir el cuerpo como morada del espíritu, como sugiere Ricardo Senabre (Tobar, 2002: 255) parece no tener aquí razón de ser; ambas, casa y cuerpo, se muestran vacías de supervivencia, como caparazones huecos de donde la vida ha huido. Sólo la naturaleza contiene en sí la eternidad, mostrándose ajena al dolor de la vejez y perpetua en la continua rueda del cambio (día-noche, hielo-deshielo). El contenido es, al fin y al cabo, lo que dota de sentido a la estructura, ya hablemos de la casa o del cuerpo, pues cuando éste falta la muerte o la ruina acechan.

Este mismo enfrentamiento del espacio marchito de la casa con el locus amoenus clásico lo encontramos en el poema "Salicio vive en el tercero izquierda" del poeta salmantino ${ }^{4}$. En este ejemplo el locus amoenus es un espa-

\footnotetext{
${ }^{4} \mathrm{Ni}$ siquiera hay lugar para que sea dulce el lamento, musical el llanto: aire claro, alta cumbre, verde valle alivian, glorifican, oxigenan las lágrimas: las hacen respirables, navegable a la luz la soledad..

Pero, decidme, aquí, que mi ventana $-y$ es suerte que no encuentre otro bostezo en la pared de enfrente, abajo un patio donde soñar la muerte nueve con ocho metros por segundoda a un jardín profanado por la prisa, a una boca de riego violentada, a un árbol flagelado por los sábados, a un puré de residuos, al reino que alquilaron los pastores que vendieron al lobo los rebaños... aquí, ¿qué abrazo cabe con qué que me consuele del difunto dolor - no hay dolor vivo:
} 
cio deseado, pero sustituido en la realidad del yo poético por el degradado espacio urbano. Ese yo poético habla desde un "aquí" que señala el espacio interno de la casa, lugar del desengaño que opone el paisaje que debiera haber - "aire claro, alta cumbre, verde valle"-y el que efectivamente encuentra - "un jardín profanado por la prisa”. El deseo de muerte, conjurado en una imagen netamente gongorina - "y es suerte que no encuentre otro bostezo/ en la pared de enfrente" - introduce la razón de esa ausencia, debida a "los pastores/ que vendieron al lobo los rebaños". Las referencias literarias constituyen los puntos de giro de este poema desde su título hasta la estrofa final, donde el poeta coronado paródicamente viaja en un ascensor símbolo de la nueva improductibilidad del movimiento, relegado a los límites de la casa.

\section{REDENCIÓN DE LA RUINA POR LA NATURALEZA}

La dimensión antropológica del horror de la ruina que introduce Miguel Casado al considerar la raíz profunda de esta imagen en el hombre va a poder redimirse en Aníbal Núñez, en algún matiz, en algún aspecto, por medio de su apertura a la vida de la naturaleza.

En un fragmento del poema "La belleza arrebata las palabras que intentan proclamarla” Aníbal Núñez recoge de la concepción romántica de la

hiere el hedor- de tu distancia?

Sólo

cabe un camino, un ápice de gloria:

llamar al ascensor, bajo el amparo

de la noche, ocultar unas tijeras

hasta la portería y, mientras pulsas

el botón de regreso, ante la luna,

ceñir con hiedra artificial la frente (Núñez 1995a: 153-54).

${ }^{5}$ De la mutilación de las estatuas

a veces surge la belleza, de los

capiteles truncados cuyo acanto

cayera en la maleza entre el acanto

-réplica en viejo mármol de un verdor sorprendido

por la primera lluvia que conoce-: posible

perfección del azar que nada tiene

que hacer para ser símbolo de todo

lo que se quiera

\section{Triste}

belleza -no la suya: nunca es triste

la piedra en su lugar, nunca fue triste 
ruina la relación entre destrucción y belleza, condensando en el primer verso la perspectiva simbólica de Juan Eduardo Cirlot, mutilación de la ruina que se hace aún más evidente cuando volvemos a encontrarnos de nuevo con la identificación ruina-cuerpo, pues la piedra ha tomado en la estatua la forma del hombre.

La aparición del acanto, además, da lugar a una nueva imagen, donde la piedra -ya estéril, despojada de función o utilidad- es recubierta por la naturaleza. La conjugación de la pérdida de la función primera con la acción balsámica del tiempo, por medio del elemento vegetal, matiza el dolor de esa pérdida -"sólo la naturaleza entrevista le devuelve ecos de su yo, que, entretanto, ha hecho masa crítica con el resto de ruinas tangibles cuyo inventario escrupulosamente realiza" (Flor, 2008: 228)-, aspecto bellamente descrito por Góngora en las Soledades: "Yacen ahora, y sus desnudas piedras/ visten piadosas yedras:/ que a rüinas y a estragos,/ sabe el tiempo hacer verdes halagos" (Góngora, 2009: 422).

Éstos "verdes halagos" indican un atemperarse del sufrimiento que la decadencia impone. En el poema de Aníbal Núñez, esta decadencia subrayada por el fragmento roto de la estatua -no sólo erosión lenta, sino destrucción llevada al extremo, donde ya se encuentran pedazos aislados del bloque original- se dulcifica con los mismos medios: "capiteles truncados cuyo acanto/ cayera en la maleza entre el acanto". La inteligente disposición estructural, con los dos "acantos" al final de verso, refuerza la idea consolatoria, pues al adjetivo "truncados" y al "cayera" que encabeza el segundo verso, creando una incertidumbre sostenida, se responde con el "acanto" final, que devuelve el equilibrio al discurso. Por ello, la tragedia del capitel desprendido se compensa con el encuentro en la tierra de un elemento con el que se identifica intensamente en nombre y-enfaticemos- en forma.

Las "piadosas yedras" y "verdes halagos" gongorinos tienen en el poeta salmantino, sin embargo, una función más activa, pues esa relación de la ruina con la naturaleza da lugar a un nuevo símbolo. El capitel roto con forma de acanto yace junto al acanto vivo del que toma su forma la piedra, oponiéndose de ese modo dos universos diferentes enfrentados: acanto-capitel-artificio humano, frente a acanto-maleza-naturaleza. El primer acanto,

la maleza en el suyo- la del símbolo...

Pues el azar que rompe la voluta, cercena gestos imperecederos... es el mismo que quiebra la hermosura de edificios de sangre (Núñez, 1995a: 175). 
erigido como memoria de lo humano en piedra para convertirlo en eterno, yace derrotado por el tiempo, convertido en artificio vano frente al segundo acanto, éste sí vivo, frágil pero perenne en el tiempo circular de la naturaleza. Ambos acantos yuxtapuestos generan dentro de ese azar que supone su situación en un mismo espacio un símbolo nuevo, que recoge las significaciones de los dos acantos por separado pero tomando también nuevos matices. El acanto de piedra verdea, como en el poema gongorino, es decir, adquiere elementos vitales de ese nuevo espacio -"maleza", "primera lluvia"que no deja de ser, como recuerda el poeta en los versos siguientes, su propio espacio original.

Esta consideración sobre el espacio original del acanto, -"nunca es triste/ la piedra en su lugar, nunca fue triste/ la maleza en el suyo"- sí va a ser netamente original ${ }^{6}$. En ella, Aníbal Núñez convierte la interpretación de la ruina en circular, pues la piedra, extraída de la naturaleza y convertida en artificio, debe volver a su estado primero. Este recorrido no completa, sin embargo, una circunferencia perfecta, en tanto que la materia ha ido sufriendo un proceso de pérdida, de difuminación de los contornos, que resta luminosidad al encuentro. Por ello es "Triste/ belleza [...] la del símbolo...", como son tristes los versos finales del soneto de Quevedo "A Roma sepultada en sus ruinas", donde la perdurabilidad -el Tíber- no puede ser celebrada en su esplendor, pues, aunque vivo, se convierte en signo doloroso del pasado. La diferencia entre ambos planteamientos radica en que la circularidad encontrada en la reunión entre naturaleza y artificio en el mismo plano en Aníbal Núñez -"capiteles truncados cuyo acanto/ cayera en la maleza entre el acanto"- es en Quevedo apenas superación postrera de lo natural que, en medio del discurrir irrefrenable del tiempo, cubre el artificio: yuxtaposición del primero frente a superposición en el segundo.

El equilibrio entre naturaleza y artificio establece en cada caso una relación cinética también divergente. Mientras en Aníbal Núñez encontramos un plano vertical, donde la ruina cae en la naturaleza, por quien es acogida y, en cierto modo, tanto redimida como revivida, Quevedo crea un eje horizontal en el que los dos elementos -agua y piedra- están en lugares contiguos de ese plano. No hay acercamiento, como en el salmantino, sino progresiva desaparición de una de las partes, que culmina en una ausencia apenas señalada por medio de indicios ("sepoltura"). Poco espacio resta para

${ }^{6}$ Como apunta Emilio Orozco, en el Barroco la belleza de las ruinas reside "en esa sensación de que lo artificial, lo artístico, se incorpora a la Naturaleza" (1947: 123), incorporación que va a diferir de esta vuelta a los orígenes propuesta por Núnez. En el primer caso la naturaleza se impone al artificio; en el segundo se reconoce en el artificio la naturaleza, convocando su unidad. 
la esperanza, pues si en Aníbal Núñez la destrucción se consuela por la vuelta al origen de la materia en medio de una naturaleza perpetua, Quevedo nos muestra cómo el tiempo altera incluso ésta última, pues si la piedra deriva en ruina para desaparecer, el río altera su función nutricia ("si ciudad la regó") volviéndose lágrima ("la llora con funesto son doliente").

La elección de la estatua, figura antropomorfa, que ve cómo una parte de sí se desprende, nos enfrenta de nuevo con el envejecimiento del hombre, doloroso y bello a la vez. La esperanza final, el reverdecer de esa materia muerta integrada ya en el tiempo cíclico de la naturaleza, equilibra el tono del poema, pese a que predomina la belleza triste de la decadencia humana -"el azar que rompe la voluta,/ cercena gestos imperecederos.../ es el mismo que quiebra la hermosura/ de edificios de sangre"-, al sufrir el cuerpo el mismo rigor del tiempo que la ruina. La aparición del dolor y la belleza juntos se entiende en Aníbal Núñez también en clave metapoética, donde la palabra es una necesidad dolorosa pero necesaria para acceder al reino de lo real: "Dolor de la palabra:/ tener que hacerse verbo para hacerse/ carne, flor, mármol:/ es del aire y las aves/ la llevan en sus alas/ y en su sangre los hombres".

El ejemplo más representativo de la unión del lamento por la ruina y la recreación en su belleza lo tenemos en la atmósfera decadente del extenso poema "Casa Lys", donde el vaivén afectivo se recrea en el exceso creando una imagen que a veces se ha descrito como ruina pródiga, en tanto que causa a partes iguales gozo y dolor ${ }^{7}$. Esta exuberancia forma parte de esa poética inclinación que recorre el alma del poeta, como constata desde su "Poética I": "Y rotos, agujeros, ojos de cerradura que susciten curiosidad y búsqueda de esa pieza que encaje. (Como barroco, amo lo truncado, el defecto o exceso que reclamen)" (Núñez, 1995b: 115).

\section{CONCLUSIONES}

Del mismo modo que Aníbal Núñez evita recoger algunos tópicos barrocos, quizá aquéllos más anclados a la retórica de la ruina, nuevos elementos surgen en paralelo con los antiguos. Entre ellos, podemos destacar el nuevo yo que contempla la ruina: frente al peregrino que aparece en el comienzo

${ }^{7}$ Ve Ángel L. Prieto de Paula en esta conjunción una de las claves actuales de la dirección que toma el tópico de la ruina: "Horra de todo didactismo, la reflexión filosófico-moral resulta hoy entronización de la belleza en sí, y de su caducidad. Una lección definitiva de nihilismo" (1991: 262). 
apelativo del soneto de Quevedo - "Buscas en Roma a Roma, ¡oh peregrino!"-, el yo poético de obras como Alzado de la Ruina o Cuarzo toma la figura del paseante que deambula por la ciudad y la provincia de Salamanca atesorando edificios y ruinas, dejando constancia en innumerables poemas de esa personal "necesidad compulsiva de recorrer y registrar el ámbito de la ciudad" (Flor, 2008: 244). Nombres propios de espacios de su realidad más íntima (la Casa Lys, el Teso de San Cristóbal, la Mansio Séntice, el Real Fuerte de la Concepción, el río Tormes) aparecen apuntalando la vivencia personal de la ruina en Aníbal Núñez. Más allá del tópico literario, ésta se inserta en la biografía del poeta configurando su espacio más estrictamente personal, aquel que es literaturizado pero también, y en este caso además con una crudeza aplastante, absolutamente vivido.

Ese punto de vista del poeta biográfico podría explicar la sustitución del espacio único del peregrino barroco por el pasear dinámico del salmantino, originalidad que redunda en la sensación de ciclicidad temporal al sustituir el espacio como meta por el deambular azaroso en el espacio urbano, donde la ruina aparece y desaparece imprevisiblemente. Incluso atendiendo a la significación propuesta por Lara Garrido del peregrino como "el hombre en la dimensión transitoria y fugitiva que refleja el devenir inexorable de su obra” (1980: 289), el contemplador en Aníbal Núñez no está enfrentado a la desaparición sino al instante de la sucesión de desapariciones y apariciones. Esa pluralidad de ruinas fuerza la mirada del poeta, que se aferra a la solidez de la ruina con una nueva resignificación de morada. Si momentos antes ésta era apenas vestigio de construcción, ahora es vivienda, aunque sea de los desechos y los desechados de la ciudad. Esta perspectiva se convierte en símbolo absoluto cuando la casa deriva en templo: "recientes moradores te ignoran, absorbida/ toda su fantasía por espacios más fáciles,/ siendo tú santuario de los que sufren cerco-/ para ellos un escollo, un peligroso signo/ de lo que no se entiende porque no se repite" (Núnez, 1995a: 251). Templo investido de sacralidad por sus propias características, no por ser vestigio de un espacio anteriormente sagrado. La supervivencia estricta que busca refugio en la ruina se transforma en conocimiento cuando los habitantes, aquellos conscientes, la convierten en vórtice. Esta cualidad sagrada de la ruina (González Gil, 2009; Puppo, 2006: 204) depende, pues, no de su estado físico sino de su carácter irrepetible en tanto que construcción del azar, pero también se construye sobre la mirada sabia de quien la habita. Como espacio sagrado, oculto, apenas reconocible, se convierte en espacio de iniciación y de acceso al conocimiento. Un conocimiento de tipo no racional, "peligroso signo/ de lo que no se entiende porque no se repite", cifrado, para 
Penélope Casado, en un hermetismo clásico, pues "al modo de los palacios y templos herméticos renacentistas, las ruinas son el espacio capaz de inducir ciertos estados de conciencia, de conectar con el cosmos" (2005: 166).

Quizá sea esta instauración de una nueva perspectiva temporal, desde la que instalarse en el presente, la gran actualización del motivo barroco de la ruina que lleva a cabo en sus poemas Aníbal Núñez, donde "INSTANTE y SITUACIÓN, las grandes palabras que en nuestro tiempo (al que la obra del poeta Aníbal Núñez apunta) surgen, deben venir a sustituir definitivamente a lo que, por un lado, ha sido la engañosa ETERNIDAD (con que habla el idealismo) y, por otro lado, la despiadada HISTORIA (de construcción desoladamente materialista)" (Flor, 2003: 12).

\section{REFERENCIAS}

Benéitez Andrés, Rosa. (2010). Sobre un re-verso de Walter Benjamin. Constelaciones: Revista de Teoría Crítica, 2, 253-260.

Casado, Miguel. (1999). La puerta azul. Madrid: Hiperión. - (2008). Belleza triste del símbolo. En Mecánica del vuelo. En torno al poeta Aníbal Núnez (pp. 67-92). Madrid: Círculo de Bellas Artes.

Casado, Penélope. (2005). Aníbal Núnez en la tradición hermética. Letras de Deusto, 35, 108, 159-76.

Cirlot, Juan Eduardo. (2007). Diccionario de símbolos. Madrid: Siruela.

Díez de Revenga, Francisco J. (2004). Más sobre la recepción de Quevedo por los poetas del siglo XX: Quevedo y Jorge Guillén. La Perinola, 8, 109-123.

Ferri Coll, José María. (2004). Itálica abolida. Una lección de vanitas en la poesía española contemporánea. Anales de literatura española, 17, 35-48. . (2010). Miré los muros de la patria mía y la tradición poética de las ruinas. Bulletin of Hispanic Studies, 87, 5, 523-544.

Flor, Fernando R. de la. (2003). Primavera soluble. En Aníbal Núñez, Primavera soluble (pp. 7-16). Salamanca: Diputación de Salamanca. (2008). La poética vital de Aníbal Núñez. En Miguel Casado (ed.), Mecánica del vuelo. En torno al poeta Aníbal Núñez (pp. 203-245). Madrid: Círculo de Bellas Artes.

García Valdés, Olvido. (2008). Quebrada, quiebro, quebranto. En Miguel Casado (ed.), Mecánica del vuelo. En torno al poeta Aníbal Núnez (pp. 25-32). Madrid: Círculo de Bellas Artes. 
Góngora, Luis de. (2009). Antología poética. Edición de Antonio Carreira. Barcelona: Crítica.

González Gil, Isabel. (2009). En la ciudad perdida: la paradoja del espacio en la poesía de Aníbal Núñez. Ángulo Recto, 1 [en línea]. Disponible en http://www.ucm.es/info/angulo/volumen/Volumen01-1/textos02.htm. Consulta: 23.10.2013.

Herrero Álvarez, Joaquín. (2005). La lírica de Aníbal Núñez. Tesis doctoral defendida en la Universidad Complutense de Madrid, España, dirigida por Mercedes López Suárez.

Labrador Méndez, Germán. (2007). Lecturas de tomo y lomo. Bibliotecas, poetas y comunidades hermenéuticas (1970-1988): El inventario de los libros de Aníbal Núñez. Anuario 2007 del Instituto de estudios zamoranos Florián de Ocampo, 24, 567-82.

Lara Garrido, José. (1980). Notas sobre la poética de las ruinas en el barroco. Analecta Malacitana, 3, 385-99.

López Bueno, Begoña. (1986). Tópica literaria y realización textual: Unas notas sobre la poesía española de las ruinas en los siglos de oro. Revista de filología española, 66, 1/2, 59-74.

Martos Pérez, María D. (2008). Las ruinas en la poesía española contemporánea. Estudio y antología. Málaga: Universidad de Málaga.

Núñez, Aníbal. (1995a). Obra Poética, I. Madrid: Hiperión. (1995b). Obra Poética, II. Madrid: Hiperión.

Orozco Díaz, Emilio. (1947). Ruinas y jardines. Su significación y valor en la temática del Barroco. En Temas del Barroco. Granada: Universidad de Granada.

Pardellas Velay, Rosamna. (2009). El arte como obsesión: la obra poética de Aníbal Núñez en el contexto de la poesía española de los años 70 y 80 . Madrid: Verbum.

Piera, Carlos. (2008). Terca piedad. En Miguel Casado (ed.), Mecánica del vuelo. En torno al poeta Aníbal Núnez (pp. 33-55). Madrid: Círculo de Bellas Artes.

Prieto de Paula, Ángel L. (1991). La tradición literaria de las ruinas en los poetas del 70. En La lira de Arión. De poesía y poetas españoles del siglo XX (pp. 221-262). Alicante: Universidad de Alicante.

Profeti, María Grazia. (2003). "Yo vi la grande y alta jerarquía". El tema de las ruinas en Quevedo. Criticón, 87-88-89, 709-18.

Puppo, María Lucía. (2006). De ruinas y cristales: una poética del tiempo en los textos de Aníbal Núñez. Revista de Literatura, 68, 135, 199-219. 
Quevedo, Francisco de. (1969). Obra poética, I. Edición de J. M. Blecua. Madrid: Castalia.

Revilla, Federico. (2007). Fundamentos antropológicos de la simbología. Madrid: Cátedra.

Rey, Alfonso. (1997). Vida retirada y reflexión sobre la muerte en ocho sonetos de Quevedo. La Perinola, 1, 189-211.

Rozas López, Juan Manuel. (1999). José-Miguel Ullán, Aníbal Núñez, Leopoldo María Panero. En Francisco Rico (ed.), Historia y crítica de la literatura española, vol. 8, No 1 (pp. 302-10). Barcelona: Crítica.

Tobar Quintanar, María José. (2002). "Miré los muros de la patria mía” y la reescritura en Quevedo. La Perinola, 6, 239-62. 